\title{
Bounded rational learning in differential information economies: core and value
}

\author{
Leonidas Koutsougeras a,b, Nicholas C. Yannelis ${ }^{\mathrm{c}, *}$ \\ ${ }^{\text {a }}$ CORE, Université Catholique Louvain-la-Neuve, Belgium \\ ${ }^{\mathrm{b}}$ Department of Economics, University of Manchester, UK \\ ${ }^{\mathrm{c}}$ Department of Economics, University of Illinois at Urbana-Champaign, Champaign, IL 61820, USA
}

Received 1 June 1997; accepted 1 September 1997

\begin{abstract}
We apply the private core and the private value to a dynamic economy and study the evolution of private core and private value allocations as individuals acquire information (learn) through time. In particular, a realized agreement in each period generates information that changes the underlying information structure in the economy. Since the private core (value) depends on the distribution of information across individuals, when agents learn some new information a realized agreement may no longer be in the private core (value) for the new information structure that arises. We define a 'limit full information' economy and address the following issues; (i) Given a sequence $x^{t}$ of approximate private core (value) allocations in each period, there is a subsequence that converges to a private core (value) $x^{*}$ allocation for the limit full information economy. (ii) Private core (value) allocations $x^{*}$ in the limit full information economy, can be approximated by a sequence $x_{e}^{t}$ of approximate private core (value) allocations in each time period. The approximate notions we consider can be viewed as the errors that agents make due to bounded rationality. (C) 1999 Elsevier Science S.A. All rights reserved.
\end{abstract}

Keywords: Private core; value allocations; Private value allocations; Dynamic economy; Approximate notions

\footnotetext{
* Corresponding author. University of Illinois, Department of Economics, 330 Commerce West, 1206 South Sixth Street, Champaign, IL 61820, USA. E-mail: nyanneli@uiuc.edu
} 


\section{Introduction}

There is an interesting growing literature on learning which mainly focuses on non-cooperative equilibrium concepts in either normal or extensive form games, e.g., Feldman (1987), Kalai and Lehrer (1993), Nyarko (1994) among others. We depart from the above literature by examining learning in differential information economies with solution concepts which may be cooperative. In particular, we abandon the rational expectations equilibrium notion and examine concepts such as the core and the Shapley value. We wish to emphasize at the outset that the study of cooperative solution concepts (e.g., the core and the value) in differential information economies appears to be a successful alternative to the traditional rational expectations equilibrium, because they provide sensible and reasonable outcomes in situations where any rational expectations equilibrium (REE) notion fails to do so. ${ }^{1}$

We begin by defining the concept of an economy with differential information [or Radner-type economy, Radner, 1968].

Let $Y=\mathbb{R}_{+}^{\ell}$ be the positive cone of $\ell$-fold Cartesian product of the set of real numbers $\mathbb{R}$ and let $(\Omega, \mathscr{F}, \mu)$ be a probability measure space. ${ }^{2}$ An exchange economy with differential information $\mathscr{E}$ is given by $\mathscr{E}=\left\{\left(X_{i}, u_{i}, \mathscr{F}_{i}, e_{i}, q_{i}\right)\right.$ : $i=1,2, \ldots, n\}$, where

1. $X_{i}: \Omega \rightarrow 2^{Y}$ is the consumption set of agent $i$,

2. $u_{i}: \Omega \times Y \rightarrow \mathbb{R}$ is the utility function of agent $i$,

3. $\mathscr{F}_{i}$ is a (finite measurable) partition of $\Omega$ denoting the private information of agent $i,{ }^{3}$

4. $e_{i}: \Omega \rightarrow Y$ is the initial endowment of agent $i$, where each $e_{i}$ is $\mathscr{F}_{i}$-measurable, (Bochner) integrable and $e_{i}(\omega) \in X_{i}(\omega) \mu$-a.e.,

5. $q_{i}: \Omega \rightarrow \mathbb{R}_{++}$is a Radon-Nikodym derivative denoting the prior of agent $i$, such that $\int q_{i}(\omega) \mathrm{d} \mu(\omega)=1$.

For each $i,(i=1,2, \ldots, n)$, denote by $E_{i}(\omega)$ the event in $\mathscr{F}_{i}$ containing the realized state of nature $\omega \in \Omega$ and suppose that $\int_{t \in E_{i}(\omega)} q_{i}(t) \mathrm{d} \mu(t)>0$ for all $i$.

\footnotetext{
${ }^{1}$ See Koutsougeras and Yannelis (1994) for examples to that effect. Recently, Kurz (1994) has also introduced an alternative to the rational expectations equilibrium. Although his viewpoint is different than ours, it also appears to provide more sensible results than the traditional rational expectations equilibrium.

${ }^{2}$ The results of this paper hold true even if $Y$ is the positive cone of a Banach lattice with an order continuous norm (see Section 5 for a precise definition). Hence infinitely, many commodities are allowed in our model.

${ }^{3}$ Throughout our analysis we follow Aumann (1987) and assume that the private information sets $\left\{\mathscr{F}_{i}\right\}_{i=1}^{n}$ are common knowledge.
} 
Given $E_{i}$ ( $\omega$ in $\mathscr{F}_{i}$ define the conditional (interim) expected utility of agent $i$, $v_{i}: \Omega \times L_{x_{i}} \rightarrow \mathbb{R}$ by ${ }^{4}$

$$
v_{i}\left(w, x_{i}\right)=\int_{t \in E_{i}(w)} u_{i}\left(t, x_{i}(t)\right) q_{i}\left(t \mid E_{i}(w)\right) \mathrm{d} \mu(t)
$$

where

$$
q_{i}\left(t \mid E_{i}(w)\right)=\left\{\begin{array}{cl}
0 & \text { if } t \notin E_{i}(w) \\
\frac{q_{i}(t)}{\int_{t \in E_{i}(w)} q_{i}(t) \mathrm{d} \mu(t)} & \text { if } t \in E_{i}(w) .
\end{array}\right.
$$

A possible interpretation of the above economy is the following: one may think that there are two periods where actual consumption takes place in the second period. In period one, there is uncertainty over the states of nature and in this period agents make agreements in an interim stage, i.e., once they are informed which is the event which contains the realized state of nature. Agents have different priors and they update them using the Bayes' rule. Once the state of nature is realized agents carry out the agreements made in the interim stage and consumption takes place.

The traditional notion which has been adopted in the literature to analyze trade in a differential information economy is the rational expectation (price) equilibrium. One of the criticisms of the above notion is that it does not provide a mechanism which describes how equilibrium prices reflect the information asymmetries of the economy. To this end we adopt the private core (Yannelis, 1991) and the private (Shapley) value (Krasa and Yannelis, 1994) in order to analyze the trading procedure under differential information. Both notions are not fully cooperative in a differential information economy framework, because within a coalition agents make redistributions of their initial endowments based on their own private information (without necessarily sharing it). Hence, despite the fact that coalitions of agents get together and make redistributions (the cooperative aspect of the model) there is a non-cooperative element in that agents in the coalition bargain using their differential information. This non-cooperative feature of the private core and private value results in allocations which are always coalitionally incentive compatible and they take into account the information superiority of an individual (contrary to the REE). ${ }^{5}$

\footnotetext{
${ }^{4}$ Note that $L_{x_{i}}=X_{i} \in L_{1}(\mu, Y): X_{i}: \Omega \rightarrow Y$ is $\mathscr{F}_{i}$-measurable and $X_{i}(\omega) \in X_{i}(\omega) \mu$-a.e. $\}$, where $L_{1}(\mu, Y)$ denotes the space of equivalence classes of $Y$-valued (Bochner) integrable functions $y$ : $\Omega \rightarrow Y$, see also Section 5.1 for a precise definition of the Bochner integral. If $Y=\mathbb{R}_{+}^{\ell}$ this is the standard Lebesgue integral.

${ }^{5}$ See Koutsougeras and Yannelis (1993) and Krasa and Yannelis (1994) for a treatment of the coalitional incentive compatibility properties of the private core and value. In a different context and prior to the above papers Myerson (1984) has analyzed individually incentive compatible cooperative solution concepts.
} 
Moreover, it has been indicated in Koutsougeras-Yannelis and Krasa-Yannelis, that the resulting private core (value) allocation will always depend on the underlying information structure. Thus, it is natural to conclude that the set of private core (or private value) allocations will be affected by changes in the underlying information structure of the economy. The concept of learning introduces changes in the information structure in a natural way. In particular, we will define a differential information economy that extends to many periods. Agents are endowed with some initial private information, drawn from their personal characteristics (i.e., their random preferences and random initial endowment). However, in each period agents acquire new information by observing the realized core (or value) allocation and they use this new information in subsequent periods. To be precise consider the differential information economy $\mathscr{E}$ in a dynamic framework.

Let the set $T=\{1,2, \ldots\}$ denote the time horizon, and denote by $\sigma\left(e_{i}^{t}, u_{i}\right)$ the $\sigma$-algebra that the random initial endowment and random utility function of agent $i$, generate at time $t$ in $T$. This is the initial information of agent $i$ at time $t$. At any given point in time $t \in T$, the private information set of agent $i$ is defined as:

$$
\mathscr{F}_{i}{ }^{t}=\sigma\left(e_{i}^{t}, u_{i}\left\{x^{t-1}, x^{t-2}, \ldots\right\}\right),
$$

where $x^{t-1}, x^{t-2}, \ldots$ are past period private core allocations. In other words, at any given point in time $t$, the private information which is available to agent $i$ is his / her initial information $\sigma\left(e_{i}^{t}, u_{i}\right)$ together with the information that private core allocations generated in all previous periods, i.e., $t-1, t-2, \ldots$ In this scenario, the private information set of agent $i$ in period $t+1$ will be $\mathscr{F}_{i}{ }^{t}$ together with the information that the private core allocation generated at period $t$, i.e., $\sigma\left(x^{t}\right)$. Thus, the private information set of agent $i$ at time $t+1$ will be $\mathscr{F}_{i}{ }^{t+1}=\mathscr{F}_{i}{ }^{t} \mathrm{~V}$ $\sigma\left(x^{t}\right)$, (where the symbol $\vee$ denotes the 'join,' i.e., $\mathscr{F}_{i}{ }^{t} \vee \sigma\left(x^{t}\right)$ is the smallest $\sigma$-algebra containing $\mathscr{F}_{i}^{t}$ and $\left.\sigma\left(x^{t}\right)\right)$. Clearly, in period $t+2$ the private information set of agent $i$ will be $\mathscr{F}_{i}{ }^{t+2}=\mathscr{F}_{i}{ }^{t+1} \vee \sigma\left(x^{t+1}\right)$ and so on. Consequently, for each player $i$ and each time period $t$ we have that

$$
\mathscr{F}_{i}{ }^{t} \subseteq \mathscr{F}_{i}{ }^{t+1} \subseteq \mathscr{F}_{i}{ }^{t+2} \subseteq \ldots
$$

Eq. (1.3) represents a learning process for agent $i$ and it generates a sequence of differential information economies $\left\{\mathscr{E}^{t}: t \in T\right\}$ defined as above where now the private information set of each agent is given by Eq. (1.2)

It is important to note that agents are myopic, in the sense that they do not form expectations over the entire horizon but only for the current period, i.e., each agent's interim expected utility is based on the current period private information. Obviously, since the private information set of each agent becomes finer from period to period (because of the acquired new information), the interim expected utility of each agent is changing from period to period as well. In this scenario the information that the private core (or value) allocation generates at a given time $t$ in $T$, obviously will affect the private core (or value) outcome in the subsequent 
periods, $t+1, t+2, \ldots$ Let us motivate this further by means of the following example.

Example 1.1: Consider the following differential information economy with two agents denoted by $K, L$ and three equally probable states denoted by $a, b, c$ (i.e., $\Omega=\{a, b, c\})$. There are two goods per state denoted by $x$ and $y$. The time horizon is denoted by $t, t+1, \ldots$ The random initial endowment and the private information set of each agent in period $t$ are as follows: ${ }^{6}$

$$
\begin{array}{ll}
e_{K}^{t}=((10,0),(10,0),(0,0)), & \mathscr{F}_{K}^{t}=\{\{a, b\}\},\{\{c\}\} \\
e_{L}^{t}=((0,10),(0,0),(0,10)), & \mathscr{F}_{L}^{t}=\{\{a, c\}\},\{\{b\}\} .
\end{array}
$$

Both agents have the same utility function given by $u_{i}(\omega, x, y)=\sqrt{x y}$, for each $\omega \in \Omega$. Suppose that nature chooses state a (which is in the event $\{a, b\}$ for agent $K$ and $\{a, c\}$ for agent $L$ ). It can be checked that in period $t$ the private core allocation is the initial endowment, ${ }^{7}$ i.e., the private core allocation in period $t$ is the vector $\left(x_{K}^{t}, x_{L}^{t}\right)=\left(e_{K}^{t}, e_{L}^{t}\right)$ and each agent gets zero utility. Notice that $\sigma\left(x_{K}^{t}, x_{L}^{t}\right)=\{\{a\},\{b\},\{c\},\{a, b\},\{b, c\},\{a, c\},\{a, b, c\}, \varnothing\}$, i.e., the information that the private core allocation generates in period $t$ is the full information partition. Therefore, the private information set of each agent in period $t+1$ will be:

$$
\begin{aligned}
& \mathscr{F}_{K}^{t+1}=\mathscr{F}_{K}^{t} \vee \sigma\left(x_{K}^{t}, x_{L}^{t}\right)=\{\{a\},\{b\},\{c\}\} \\
& \mathscr{F}_{L}^{t+1}=\mathscr{F}_{L}^{t} \vee \sigma\left(x_{K}^{t}, x_{L}^{t}\right)=\{\{a\},\{b\},\{c\}\} .
\end{aligned}
$$

Now in the second period, agents will make contracts based on the private information sets given by Eq. (1.5). One can show that the private core allocation in period $t+1$ is the following:

$$
\begin{aligned}
& x_{K}^{t+1}=((5,5),(10,0)(0,0)) \\
& x_{L}^{t+1}=((5,5),(0,0),(0,10)) .
\end{aligned}
$$

Notice that the above allocation makes both agents better off from the allocation given by Eq. (1.4) (they both now have positive utility). In other words, both agents by observing the private core allocation in period $t$ and refining their private information they result in a Pareto superior allocation.

\footnotetext{
${ }^{6}$ In other words the endowment pattern is as follows:

$\begin{array}{llll} & \mathrm{a} & \mathrm{b} & \mathrm{c} \\ \mathrm{K} & (10,0) & (10,0) & (0,0) \\ \mathrm{L} & (0,10) & (0,0) & (0,10)\end{array}$

${ }^{7}$ Since the partitions are independent, agents cannot insure each other and no trade is the equilibrium outcome in period $t$. See also definition 2.1 for a rigorous definition of the private core.
} 
In the above simple example, learning enabled the two agents to reach the limit full information private core in period $t+1$ where no further trade improvement is possible. However, in a general model with more than two agents and a continuum of states there is no need to reach the limit full information private core outcome in two periods. Our main objective in this paper is to examine the convergence of the private core (or private value) of the infinitely repeated differential information economy. In particular, let us denote the one shot limit full information economy ${ }^{8}$ by $\left.\overline{\mathscr{E}}=\left(X_{i}, u_{i}, \overline{\mathscr{F}}_{i}, \overline{\mathrm{e}}_{i}, q_{i}\right): i=1,2, \ldots, n\right\}$ where $\overline{\mathscr{F}}_{i}$ is the pooled information of agent $i$ over the entire horizon, i.e., $\overline{\mathscr{F}}_{i}=\vee_{t=1}^{\infty} \overline{\mathscr{F}}_{i}^{t}$ and $\overline{\mathrm{e}}_{i}$ is $\overline{\mathscr{F}}_{i}$-measurable.

The questions that this paper addresses are the following.

(i) If $\left\{\mathscr{E}^{t}: t \in T\right\}$ is a sequence of differential information economies and $x^{t}$ is a sequence of private core (or value) allocation for $\mathscr{E}^{t}$ can we extract a subsequence which converges to a limit full information private core (or value) allocation for the limit full information economy $\overline{\mathscr{E}}$ ?

(ii) Is question $(i)$ still true if we allow for bounded rationality in the sense that the sequence $x^{t}$ is now an approximate or $\epsilon$-private core (or $\epsilon$-value) allocation for $\mathscr{E}^{t}$, but nonetheless it converges to an exact private core (or value) allocation?

(iii) Given a limit full information private core (or value) allocation say $\bar{x}$ for $\overline{\mathscr{E}}$ can we construct a sequence of approximate or $\epsilon$-private core (or $\epsilon$-value) allocations $x^{t}$ in $\mathscr{E}^{t}$ which converges to the limit full information private core (or value) allocation $\overline{\mathrm{x}}$ of $\mathscr{E}$ ? In other words, given a limit full information private core (or value) allocation can we construct a sequence of bounded rational plays (i.e., $\epsilon$-private core or $\epsilon$-private value allocations) which converges to the limit full information private core (value) allocation.

We indeed provide positive answers to all the above questions. It should be noted that in this paper not only do we address learning in differential information economies adopting cooperative solution concepts for the first time, but we also make several technical advances. In particular, we will allow for continuous time, i.e., the time horizon set $T$, may be any arbitrary infinite set, utility functions are concave (hence risk aversion is allowed) agents are allowed to be bounded rational during the learning process and the state space and the commodity space need not be finite. The above generalization necessitates the use of functional and measure analytic methods.

Finally, it should be noted that since our framework is quite general, it may be the case that in the limit incomplete information may still prevail. In other words, it could be the case that

$$
\overline{\mathscr{F}}_{i}=\bigvee_{t=1}^{\infty} \mathscr{F}_{i}{ }^{t} \subset \bigvee_{i=1}^{n} \mathscr{F}_{i} .
$$

Hence, in the limit a private core (or value) allocation may not be a fully revealing

\footnotetext{
${ }^{8}$ An alternative terminology may be maximal information economy.
} 
core (or value) allocation. However, if the learning through the private core (value) allocation of each period reaches the complete information in the limit, i.e.,

$$
\overline{\mathscr{F}}_{i} \supset \bigvee_{i=1}^{n} \mathscr{F}_{i},
$$

then our first converge result indicates that the private core (value) allocation is indeed a fully revealing core (value) allocation.

The paper proceeds as follows: In Section 2, we provide definitions of the private and approximate private core. Sections 3 and 4 contain the main results and all the proofs are collected in Section 5.

\section{The private core and the $\epsilon$-private core}

Before we state our main results for the private core we will redefine below the private core in a more convenient way.

For each $i$, let $L_{x_{i}}$ denote the set of all (Bochner) integrable and $\mathscr{F}_{i}$-measurable selections from the consumption set $X_{i}$ of agent $i$. Set $L_{x}=\prod_{i=1}^{n} L_{x_{i}}$. We can now restate definition 1.1 in a more convenient way as follows:

Definition 2.1: The allocation $x \in L_{x}$ is said to be a private core allocation for $\mathscr{E}$ if:

(i) $\sum_{i=1}^{n} x_{i}=\sum_{i=1}^{n} e_{i}$ and

(ii) it is not true that there exist coalition $S$ and $y \in \prod_{i \in S} L_{x_{i}}$ such that $\sum_{i \epsilon S} y_{i}=\sum_{i \epsilon S} e_{i}$ and $v_{i}\left(\omega, y_{i}\right)>v_{i}\left(\omega, x_{i}\right) \mu$-a.e., and for all $i \in S$.

Note that since the initial endowment of each agent is measurable with respect to his /her own partition and each vector $x_{i}(\mathbf{O})$ is $\mathscr{F}_{i}$-measurable, the net trades are always $\mathscr{F}_{i}$-measurable.

Definition 2.2: The allocation $x \in L_{x}$ is said to be an approximate or $\epsilon$-private core allocation for $\mathscr{E}$ if in addition to (i) above it satisfies

(ii') it is not true that there exist coalition $S$ and $y \in \prod_{i \in S} L_{x_{i}}$ such that $\sum_{i \epsilon S} y_{i}=\sum_{i \epsilon S} e_{i}$ and $v_{i}\left(\omega, y_{i}\right)>v_{i}\left(\omega, x_{i}\right)+\epsilon \mu$-a.e. and for all $i \in S$.

Condition (ii') in definition 2.1 is almost identical to condition (ii') in definition 2.2 , except that it requires the dominance of a contending allocation to be stronger. 
One may think of $\epsilon$ (which is a small positive number) as the cost of coalition formation, although a wide variety of interpretations (involving agents' reluctance to join a coalition) may also apply. Denote by $C(\mathscr{E})$ the set of all private core allocations for $\mathscr{E}$, and by $C_{\epsilon}(\mathscr{E})$ the set of $\epsilon$-private core allocations for $\mathscr{E}$.

If the exchange economy with differential information $\mathscr{E}$ satisfies for each agent $i$ the following assumptions:

Assumption a.2.1: $X_{i}: \Omega \rightarrow 2^{Y}$ is a convex, closed, non-empty valued and $\mathscr{F}_{i}$-measurable correspondence. ${ }^{9}$

Assumption a.2.2: $u_{i}: \Omega \times Y \rightarrow \mathbb{R}$ is norm continuous, integrably bounded and concave, then a private core allocation exists in $\mathscr{E}$ (see Yannelis, 1991; Koutsougeras and Yannelis, 1993).

\section{Convergence and approximation theorems for the private core and $\epsilon$-private core}

\subsection{Learning}

Let $T$ be any infinite set denoting the time horizon. Denote by $\sigma\left(e_{i}^{t}, u_{i}\right)$ the $\sigma$-algebra that the random initial endowment and utility function of agent $i$ generate at time $t$. Obviously, this is interpreted as the initial information of agent $i$ at time $t$. However, the private information which is available to agent $i$ at any given period $t$ is not only $\sigma\left(e_{i}^{t}, u_{i}\right)$ but also the information that he/she has acquired from past period private core allocations, denoted by $x^{t^{\prime}}, t^{\prime}<t, t, t^{\prime}$ in $T$. Hence, the private information set of agent $i$ at time $t$, denoted by $\mathscr{F}_{i}{ }^{t}$ is defined as:

$$
\mathscr{F}_{i}^{t}=\sigma\left(e_{i}^{t}, u_{i},\left\{x^{t^{\prime}}, t^{\prime}<t\right\}\right) .
$$

Eq. (3.1) indicates what agent $i$ has learned from past experience. Note that the private information of agent $i$ at period $t^{0}>t$ will be $\mathscr{F}_{i}{ }^{0}=\mathscr{F}_{i}{ }^{t} \vee \sigma\left(\left\{x^{t}\right.\right.$ : $\left.t \leq \bar{t}<t^{0}\right\}$ ) (where $\mathscr{F}_{i}{ }^{t} \vee \sigma\left(\left\{x^{\bar{t}}: t \leq \bar{t}<t^{0}\right\}\right.$ ) denotes the 'join,' i.e., the smallest $\sigma$-algebra containing $\mathscr{F}_{i}{ }^{t}$ and $\sigma\left(\left\{x^{\bar{t}}: t \leq^{\bar{t}}<t^{0}\right\}\right)$. Therefore for each agent $i$ and each time period $t$ we have that:

$$
\mathscr{F}_{i}{ }^{\bar{t}} \subseteq \mathscr{F}_{i}{ }^{0} \text { for } \bar{t} \leq t^{0}, \bar{t}, t^{0} \text { in } T \text {. }
$$

Eq. (3.2) represents a learning process for agent $i$.

\footnotetext{
${ }^{9}$ By $\mathscr{F}_{i}$-measurable we mean that the graph of $x_{i}$, i.e., $G_{x_{i}}=(\omega, x) \in \Omega \times Y: x \in X_{i}(\omega)$ is an element of $\mathscr{F} \otimes B(Y)$, where $B(Y)$ denotes that Borel $\sigma$-algebra on $Y$ and $\otimes$ denotes the product $\sigma$-algebra.
} 
A learning process generates a sequence of differential information economies $\left\{\mathscr{E}^{t}: t \in T\right\}$, where $\left.\mathscr{E}^{t}=\left\{X_{i}, u_{i}, e_{i}^{t}, \mathscr{F}_{i}{ }^{t}, q_{i}\right): i=1,2, \ldots, n\right\}$ is defined as in Section 1.

\subsection{The limit full information economy}

Let $\overline{\mathscr{F}}_{i}$ be the pooled information of agent $i$ over the entire time horizon $T$, i.e., $\overline{\mathscr{F}}_{i}=\bigvee_{\mathrm{t} \in \mathrm{T}} \overline{\mathscr{F}}_{i}^{t}$. The differential information economy $\overline{\mathscr{E}}=\left\{\left(X_{i}, u_{i}, \overline{\mathrm{e}}_{i}, \overline{\mathscr{F}}_{i}, q_{i}\right)\right.$ : $i=1,2, \ldots, n\}$ represents an economy where the private information of each agent, i.e., $\overline{\mathscr{F}}_{i}$ is his/her pooled information over the entire time horizon, and may be thought of as a limit full information economy. In the economy $\overline{\mathscr{E}}, X_{i}, u_{i}$ and $q_{i}$ are defined as before but now each $\bar{e}_{i}$ is $\overline{\mathscr{F}}_{i}$-measurable and Bochner integrable. Note that since in the sequel we will assume that for each $i,(i=1,2, \ldots, n)$, $\left\{e_{i}^{t}, \mathscr{F}_{i}{ }_{i}^{t}\right\}_{t \in T}$ is a martingale, it follows that $e_{i}^{t}(\omega)$, converges $\mu$-a.e. to $\bar{e}_{i}$. Hence, the initial endowment of agent $i$ in the limit full information economy, i.e., $\bar{e}_{i}$ may be viewed as the limit of $e_{i}^{t}$, and consequently each $\bar{e}_{i}$ will be $\overline{\mathscr{F}}_{i}$-measurable. By replacing in definition 2.1.1 $\mathscr{F}_{i}$ by $\overline{\mathscr{F}}_{i}$ one can obtain the notion of limit full information core allocation for the economy $\overline{\mathscr{E}}$. Denote by $C(\overline{\mathscr{E}})$ the set of all limit full information core allocations for the economy $\overline{\mathscr{E}}$.

Throughout our analysis we will assume that the net of private information economies $\left\{\mathscr{E}_{t}: t \in T\right\}$ as well as the limit full information economy $\overline{\mathscr{E}}$, satisfies the assumptions a.2.1 and a.2.2 and therefore for each $t \in T, C\left(\mathscr{E}^{t}\right) \neq \varnothing$ and $C(\overline{\mathscr{E}}) \neq \varnothing$. Moreover, since $C\left(\mathscr{E}^{t}\right) \subset C_{\epsilon}\left(\mathscr{E}^{t}\right)$ the latter set is non-empty as well. Finally, we assume that $E\left[\sum_{i=1}^{n} \bar{e}_{i} \mid \wedge_{i=1}^{n} \mathscr{F}_{i}\right]=\sum_{i=1}^{n} e_{i}^{t}$. This will guarantee the $L_{1}(\mu, Y)$-norm convergence of the aggregate initial endowment.

\subsection{Theorems}

Theorem 3.3.1: Let $\left\{\mathscr{E}^{t}: t \in T\right\}$ be a net of private information economies satisfying the following assumption:

(a.3.2) for any coalition $S,\left\{\sum_{i \in S} e_{i}^{t}, \wedge_{i \in S} \mathscr{F}_{i}^{t}\right\}_{t \in T}$ is a martingale. If the net $\left\{x^{t}: t \in T\right\}$ belongs to $C_{\epsilon}\left(\mathscr{E}^{t}\right)$ then we can extract a sequence $\left\{x^{t^{m}}\right.$ : $m=1,2, \ldots$ from the net $x^{t}$ which converges (weakly) to $x^{*} \in C(\overline{\mathscr{E}})$.

Theorem 3.3.2: Let $\left\{\mathscr{E}^{t}: t \in T\right\}$ be a net of private information economies satisfying

(a.3.3) for each $i,(i=1,2, \ldots, n),\left\{e_{i}^{t}, \mathscr{F}_{i}{ }^{t}\right\}_{t \in T}$ is a martingale.

(a.3.4) $\left\{\sum_{i=1}^{n} e_{i}^{t}, \wedge_{i=1}^{n} \mathscr{F}_{i}{ }^{t}\right\}_{t \in T}$ is a martingale.

Let $x^{*}$ be a limit full information core allocation for the economy $\overline{\mathscr{E}}$, i.e., $x^{*} \in$ $C(\overline{\mathscr{E}})$. Then, there exists a net of allocations $\left\{x^{t}: t \in T\right\}$ such that $x^{t} \in C_{\varepsilon}\left(\mathscr{E}^{t}\right)$ and $x^{t}$ converges (in the $L_{I}(\mu, Y)$-norm) to $x^{*}$.

An immediate conclusion of theorem 3.3.1 is the following result. 
Corollary 3.3.1: Let $\left\{\mathscr{E}^{t}: t \in T\right\}$ be a net of private information economies satisfying assumption 3.2. If the net $\left\{x^{t}: t \in T\right\}$ belongs to $C\left(\mathscr{E}^{t}\right)$ then we can extract a sequence from the net $x^{t}$ which converges weakly to $x^{*} \in C(\overline{\mathscr{E}})$.

\subsection{Discussion}

Assumptions 3.3 and 3.4 together imply a type of consistency of individual and aggregate expectations that we discuss below. In particular, assumption 3.4 implies that

$$
\begin{aligned}
\sum_{i=1}^{n} e_{i}^{t} & =\quad E\left[\sum_{i=1}^{n} e_{i}^{t+1} \mid \bigwedge_{j=1}^{n} \mathscr{F}_{j}{ }^{t}\right] \\
& =\sum_{i=1}^{n} E\left[e_{i}^{t+1} \mid \bigwedge_{j=1}^{n} \mathscr{F}_{j}{ }^{t}\right] .
\end{aligned}
$$

Since by assumption a.3.3, $e_{i}^{t}=E\left[e_{i}^{t+1} \mathscr{F}_{i}^{t}\right]$ by substituting this expression in the left-hand side of the above equation we obtain that

$$
\begin{aligned}
& \sum_{i=1}^{n} E\left[e_{i}^{t+1} \mid \mathscr{F}_{i}{ }^{t}\right]=\sum_{i=1}^{n} E\left[e_{i}^{t+1} \mid \bigwedge_{j=1}^{n} \mathscr{F}_{j}{ }_{j}\right] \text { or } \\
& \sum_{i=1}^{n}\left(E\left[e_{i}^{t+1} \mid \mathscr{F}_{i}{ }^{t}\right]-E\left[e_{i}^{t+1} \mid \bigwedge_{j=1}^{n} \mathscr{F}_{j}{ }^{t}\right]\right)=0 .
\end{aligned}
$$

The above expression implies that private expectations, i.e., what an individual believes his/her initial endowment will be in period $t+1$, (which is given by $E\left[e_{i}^{t+1} \mathscr{F}_{i}^{t}\right]$ ) and common knowledge expectations, i.e., what the common belief of all agents about agent $i$ 's initial endowment at period $t+1$ is, (which is given by $\left.E\left[e_{i}^{t+1} \mid \wedge{ }_{j=1}^{n} \mathscr{F}_{j}{ }^{t}\right]\right)$, must balance out on aggregate. Similarly it follows from assumption a.3.2 that for each coalition $S$,

$$
\sum_{i \in S}\left(E\left[e_{i}^{t+1} \mid \mathscr{F}_{i}^{t}\right]-E\left[e_{i}^{t+1} \mid \bigwedge_{j \in S} \mathscr{F}_{j}{ }^{t}\right]\right)=0,
$$

i.e., private expectations and coalitional common knowledge expectations ${ }^{10}$ must cancel out coalitionally.

Notice that assumption a.3.2 is equivalent to the fact that the information that the aggregate initial endowment that the coalition $S$ generates, must be common knowledge to its members, i.e., for each $t, \sigma\left(\sum_{i \in S} e_{i}^{t} \subset \wedge_{i \in S} \mathscr{F}_{i}{ }^{t}\right.$. (For a proof of this statement see lemma 5.1.)

In view of assumptions a.3.3 and a.3.4, theorem 3.3.2 shows that approximate private core allocations have the martingale property. As a consequence of this,

\footnotetext{
${ }^{10}$ By this we mean that the expression $E\left[e_{i}^{t+1} \mid \wedge{ }_{j \in S} \mathscr{F}_{j}^{t}\right]$ indicates what the belief of coalition $S$ is about the initial endowment of agent $i$ at period $t+1$.
} 
any limit full information private core allocation (i.e., any $x^{*} \in C(\overline{\mathscr{E}})$ can be reached by a net of approximate private core allocations. One may view this as a kind of stability property that the private core enjoys, in the sense that we can always find an allocation process which leads to the limit full information private core. Alternatively, one may view the conclusion of theorem 3.3.2 as a 'kind' of lower-semicontinuity (l.s.c.) of the private core and the conclusion of theorem 3.3.1 as a kind of upper-semicontinuity (u.s.c.). ${ }^{11}$ It is important however to note that we cannot speak about 1.s.c. or u.s.c. of the private core in a rigorous way because the net of differential information economies $\left\{\mathscr{E}^{t}: t \in T\right\}$ we consider need not converge (in a certain topological sense) to the limit full information economy $\overline{\mathscr{E}} .{ }^{12}$ In particular, this would only happen if one topologizes appropriately the space of utility functions and the private information sets. Although in principle this can be done we have not pursued this here because we will complicate significantly the technical apparatus of the modeling without advancing the economic insights any further.

\section{The private value allocation}

We now define the notion of a private value allocation (see Krasa and Yannelis, 1994).

Definition 4.1.1: A game with side-payments $\Gamma=(I, V)$, consists of a finite set of agents $I=\{1, \ldots, n\}$ and a superadditive, real valued function $V$ defined on $2^{I}$ such that $V(\varnothing)=0$. Each $S \subset I$ is called a coalition and $V(S)$ is the 'worth' of the coalition $S$.

The Shapley value of the game $\Gamma$, (Shapley, 1953) is a rule which assigns to each agent $i$ a 'payoff' $\mathrm{Sh}_{i}$ given by the formula

$$
S h_{i}(V)=\sum_{\substack{S \subset I \\ S \supset\{i\}}} \frac{(|S|-1) !(|I|-|S|) !}{|I| !}[V(S)-V(S \backslash\{i\})] .
$$

For each economy with differential information $\mathscr{E}$ and each set of non-negative

\footnotetext{
${ }^{11}$ Gale (1992) studies the stability of equilibrium for an incomplete information model. Roughly speaking it amounts to the fact that the equilibrium will not change very much in response to small perturbations. In our setting, one may also view our 'stability' as changes in the information sets will not change the core outcome too much.

${ }^{12}$ The u.s.c. of the private core in a rigorous setting will mean that if $\mathscr{E}^{t}$ converges (in a certain sense) to $\overline{\mathscr{E}}$ written as $\overline{\mathscr{E}}^{t} \rightarrow \overline{\mathscr{E}}$ and $x^{t}$ converges (weakly) to $x$ where $x^{t} \in C\left(\overline{\mathscr{E}}^{t}\right)$, then $x \in\left(C \overline{\mathscr{E}}^{\circ}\right)$. Similarly 1.s.c. will mean that if $\mathscr{E}^{t} \rightarrow \overline{\mathscr{E}}$ and $x \in C(\overline{\mathscr{E}})$ then there exists $\left\{x^{t}: t \in T\right\}$ such that $x^{t}$ converges to $x$ and $x^{t} \in C\left(\mathscr{E}^{t}\right)$. In a different setting and for a deterministic economy with a continuum of agents, Grodal (1971) has examined the upper-semicontinuity of the core correspondence.
} 
weights (not all equal to zero), $\left\{\lambda_{i}: i=1, \ldots, n\right\}$, we associate a game with side-payments $\left(I, V_{\lambda}\right)$ according to the rule: For $S \subset I$ let

$$
V_{\lambda}(w, S)=\max _{x_{i}} \sum_{i \in S} \lambda_{i} v_{i}\left(w, x_{i}\right)
$$

subject to

(i) $\sum_{i \in S} x_{i}(w)=\sum_{i \in S} e_{i}(w), \mu$ - a.e.

(ii) $x_{i}-e_{i}$ is $\mathscr{F}_{i}-$ measurable for every $i \in S$.

Definition 4.1.2: An allocation $x \in L_{x}$ is said to be a private value allocation of the economy with differential information $\mathscr{E}$ if the following holds:

(i) Each net-trade $x_{i}-e_{i}$ is $\mathscr{F}_{i}$-measurable.

(ii) $\sum_{i=1}^{n} x_{i}(w)=\sum_{i=1}^{n} e_{i}(w), \mu$-a.e.

(iii) There exist $\lambda_{i} \geq 0,(i=1,2, \ldots, n)$ which are not all equal to zero, with $\lambda_{i}$ $v_{i}\left(\omega, x_{i}\right)=\operatorname{Sh}_{i}\left(V_{\lambda}\right)$ for every $i$, where $\operatorname{Sh}_{i}\left(V_{\lambda}\right)$ is the Shapley value of agent $i$ derived from the game $\left(I, V_{\lambda}\right)$, defined in Eq. (4.1).

Condition (i) is equivalent to the $\mathscr{E}_{i}$-measurability of $x_{i}$ and it was discussed in Section 2 together with condition (ii). Condition (iii) says that the interim expected utility of each agent multiplied with his/her weight $\lambda_{i}$ must be equal to his /her Shapley value derived from the side-payment game $\left(I, V_{\lambda}\right)$.

An allocation $x \in L_{x}$ is said to be an approximate or $\epsilon$-private value allocation for $\mathscr{E}$ if in addition to conditions (i) and (ii) of definition 4.1.2, we have (iii) there exist $\lambda_{i} \geq 0,(i=1,2, \ldots, n)$ which are not all equal to zero such that $\lambda_{i} v_{i}\left(\omega, x_{i}\right)$ $-\operatorname{Sh}_{i}\left(V_{\lambda}\right)<\epsilon$ for all $i$, where $\operatorname{Sh}_{i}\left(V_{\lambda}\right)$ is the Shapley value of agent $i$ derived from the game $\left(I, V_{\lambda}\right)$ defined in Eq. (4.1)

Denote by $\operatorname{VA}(\mathscr{E})$ the set of all private value allocations for the economy $\mathscr{E}$. Denote by $\operatorname{VA}_{\epsilon}(\mathscr{E})$ the set of all approximate private value allocations for the economy $\mathscr{E}$. Assumptions a.2.1 and a.2.2 assure that $\mathrm{VA}(\mathscr{E}) \neq \varnothing$ (see Krasa and Yannelis, 1996). Since $\operatorname{VA}(\mathscr{E}) \subset \mathrm{VA}_{\epsilon}(\mathscr{E})$ it follows that $\mathrm{VA}_{\epsilon} \mathscr{E} \neq \varnothing$.

We can state the analogues of theorems 3.3.1, 3.3.2 and corollary 3.3.1 for the private value.

Denote by $\operatorname{VA}(\overline{\mathscr{E}})$ the set of all limit full information value allocations for $\overline{\mathscr{E}}$. It follows from assumptions 2.1 and 2.2 that $\operatorname{VA}(\overline{\mathscr{E}}) \neq \varnothing$.

We now state the convergence and approximation results for the private value allocation.

Theorem 4.2.1: Let $\left\{\mathscr{E}^{t}: t \in T\right\}$ be a net of private information economies satisfying assumption 3.3 and 3.4 of theorem 3.3 .2 and let $x^{*} \in \operatorname{VA}(\overline{\mathscr{E}})$. Then there exists a net of allocations $\left\{x^{t}: t \in T\right\}$ such that $x^{t} \in V A_{\epsilon}\left(\mathscr{E}^{t}\right.$ and $x^{t}$ converges (in the $L_{1}(\mu, Y)$-norm) to $x^{*}$. 
Theorem 4.2.2: Let $\left\{\mathscr{E}^{t}: t \in T\right\}$ be a net of private information economies satisfying the assumption 3.2 of theorem 3.3.1. If the net of allocations $\left\{x^{t}: t \in T\right\}$ belongs to $V A_{\epsilon}\left(\mathscr{E}^{t}\right)$, then we can extract a sequence from the net $\left\{x^{t}: t \in T\right\}$ which converges (weakly) to $x^{*} \in V A(\overline{\mathscr{E}})$.

Corollary 4.2.1: Let $\left\{\mathscr{E}^{t}: t \in T\right\}$ be a net of private information economies satisfying the assumption 3.2 of theorem 3.3.1. If the net $\left\{x^{t}: t \in T\right\}$ belongs to $V A\left(\mathscr{E}^{t}\right)$, then we can extract a sequence from the net $\left\{x^{t}: t \in T\right\}$ which converges weakly to $x^{*} \in V A(\overline{\mathscr{E}})$.

The interpretation of theorems 4.2.1, 4.2.2 and corollary 4.2.1 is similar to the corresponding results for the core and the discussion in Section 3.4 also applies here.

\section{Proof of the theorems}

\subsection{Mathematical preliminaries}

Let $(T, \tau, \mu)$ be a finite measure space and $X$ be a Banach space. Following Diestel and Uhl (1977) the function $f: T \rightarrow X$ is called simple if there exists $x_{1}, x_{2}, \ldots, x_{n}$ in $X$ and $\alpha_{1}, \alpha_{2}, \ldots, \alpha_{n}$ in $\tau$ such that $f=\sum_{i=1}^{n} x_{i} \chi_{\alpha_{i}}^{\prime}$, where $\chi_{\alpha_{i}}$ $(t)=1$ if $t \notin \alpha_{\mathrm{i}}$ and $\chi_{\alpha_{i}}(t)=0$ if $t \notin \alpha_{\mathrm{i}}$. A function $f: T \rightarrow X$ is said to be $\mu$-measurable if there exists a sequence of simple functions $f_{n}: T \rightarrow X$ such that $\lim _{n \rightarrow \infty} \int_{T} f_{n}(t)-f(t)=0$ for almost all $t \in T$. A $\mu$-measurable function $f$ : $T \rightarrow X$ is said to be Bochner integrable if there exists a sequence of simple functions $\left\{f_{n}: n=1,2, \ldots\right\}$ such that

$$
\lim _{n \rightarrow \infty} \int_{T}\left|f_{n}(t)-f(t)\right| \mathrm{d} \mu(t)=0 .
$$

In this case we define for each $E \in \tau$ the integral to be $\int_{E} f(t) \mathrm{d} \mu(t)=\lim _{n \rightarrow \infty} \int E$ $f_{n}(t) \mathrm{d} \mu(t)$. It can be shown (see Diestel and Uhl, 1977, theorem 2, p. 45) that, if $f: T \rightarrow X$ is a $\mu$-measurable function then $f$ is Bochner integrable if and only if $\int_{T}|f(t)| \mathrm{d} \mu(t)<\infty$. It is important to note that the Dominated Convergence Theorem holds for Bochner integrable functions, in particular, if $f_{n}: T \rightarrow X$, $(n=1,2, \ldots)$ is a sequence of Bochner integrable functions such that $\lim _{n \rightarrow \infty}$ $f_{n}(t)=f(t) \mu$-a.e., and $\left|f_{n}(t)\right| \leq g(t) \mu$-a.e., (where $g: T \rightarrow \mathbb{R}$ is an integrable function), then $f$ is Bochner integrable and $\lim _{n \rightarrow \infty} \int_{T}\left|f_{n}(t)-f(t)\right| \mathrm{d} \mu(t)=0$.

For $1 \leq p<\infty$, we denote by $L_{p}(\mu, X)$ the space of equivalence classes of $X$-valued Bochner integrable functions $x: T \rightarrow X$ normed by

$$
|x|_{p}=\left(\int_{T}|x(t)|^{p} \mathrm{~d} \mu(t)\right)^{\frac{1}{p}} .
$$

It is a standard result that normed by the functional $\|\cdot\|_{p}$ above, $L_{p}(\mu, X)$ becomes a Banach space (see Diestel and Uhl, 1977, p. 50). 
We will close this section by collecting some basic results on Banach lattices (for an excellent treatment see Aliprantis and Burkinshaw, 1985). Recall that a Banach lattice is a Banach space $L$ equipped with an order relation $\geq$ (i.e., $\geq$ is a reflexive, anti-symmetric and transitive relation) satisfying:

1. $x \geq y$ implies $x+z \geq y+z$ for every $z$ in $L$,

2. $x \geq y$ implies $\lambda x \geq \lambda y$ for all $\lambda \geq 0$,

3. for all $x, y$ in $L$ there exists a supremum (least upper bound) $x \vee y$ and an infimum (greatest lower bound) $x \wedge y$,

4. $x \geq y$ implies $\|x\| \geq\|y\|$ for all $x, y$ in $L$.

As usual $x^{+}=x \vee 0, x^{-}=(-x) \vee 0$ and $x=x \vee(-x)=x^{+}+x^{-}$; we call $x^{+}, x^{-}$the positive and negative parts of $x$, respectively and $x$ the absolute value of $x$. The symbol $\|\cdot\|$ denotes the norm on $L$. If $x, y$ are elements of the Banach lattice $L$, then we define the order interval $[x, y]$ as follows:

$$
[x, y]=\{z \in L: x \leq z \leq y\} .
$$

Note that $[x, y]$ is norm closed and convex (hence weakly closed). A Banach lattice $L$ is said to have an order continuous norm if, $x_{\alpha} \downarrow 0$ in $L$ implies $x_{\alpha} \downarrow 0 .{ }^{13}$ A very useful result which is going to play an important role in the sequel is that if $L$ is a Banach lattice then the fact that $L$ has order continuous norm is equivalent to the weak compactness of the order interval $[x, z]=\{y \in L: x \leq y \leq z\}$ for every $x, z$ in $L$ (see for instance Aliprantis and Burkinshaw, 1989, theorem 2.3.8). Moreover, Cartwright's theorem (Cartwright, 1974) asserts that if $X$ is a Banach lattice with an order continuous norm, then $L_{1}(\mu, X)$ has order continuous norm (i.e., order intervals in $L_{1}(\mu, X)$ are weakly compact). This theorem will be used for the case where $X=\mathbb{R}^{\prime}$.

Recall that if $(T, \tau, \mu)$ is a finite measure space, a sub- $\sigma$-field of $\tau$ is a subset of $\tau$ that contains $T$ and that is a $\sigma$-field itself. Let $A$ be a sub- $\sigma$-field of $\tau$ and $x$ be an element of $L_{1}(\mu, X)$. We say that $g \in L_{1}(\mu, X)$ is the conditional expectation of $x$ relative to $A$ (denoted by $E(x A))$ if $g$ is $A$-measurable and

$$
\int_{B} g(t) \mathrm{d} \mu(t)=\int_{B} x(t) \mathrm{d} \mu(t) \text { for all } B \text { in } A .
$$

We close this section by defining the notion of a martingale and stating the martingale convergence theorem.

Let $I$ be a directed set, and let $\left\{\mathscr{F}_{t}: t \in I\right\}$ be a monotone increasing net of sub- $\sigma$-fields of $\tau$ (i.e., $\mathscr{F}_{t_{1}} \subseteq \mathscr{F}_{t_{2}}$ for $t_{1} \leq t_{2}, t_{1}, t_{2}$ in $I$ ). A net $\left\{x_{t}: t \in I\right\}$ in $L_{p}(\mu, X),(1 \leq p<\infty)$ is a martingale if

$$
E\left(x_{t} \mid \mathscr{F}_{t_{1}}\right)=x_{t_{1}} \text { for all } t \geq t_{1} \text {. }
$$

We will denote the above martingale by $\left\{x_{t}, \mathscr{F}_{t}\right\}_{t \in I}$. The following convergence result will be used in some of the proofs. A martingale $\left\{x_{t}, \mathscr{F}_{t}\right\}_{t \in I}$ in $L_{p}(\mu, X)$, $(1 \leq p<\infty)$ converges in the $L_{p}(\mu, X)$-norm if and only if there exists $x$ in

\footnotetext{
${ }^{13} x_{\alpha} \downarrow 0$ means that $x_{\alpha}$ is a decreasing net with $\inf _{\alpha} x_{\alpha}=0$.
} 
$L_{p}(\mu, X)$ such that $E\left(x \mathscr{F}_{t}\right)=x_{t}$ for all $t \in I$. Note that if the martingale $\left\{x_{t}, \mathscr{F}_{t}\right\}_{t \in I}$ converges in the $L_{1}(\mu, X)$-norm, it also converges almost everywhere, i.e., $\lim _{t \rightarrow \infty} x_{t}=x$ almost everywhere (Diestel and Uhl, 1977, p. 49).

With all these preliminaries out of the way we can now turn to the proofs of our theorems.

Proof of theorem 3.3.1: We begin with some preliminary observations. For each $i,(i=1,2, \ldots, n)$ let $\bar{L}_{x_{i}}$ be the set $\left\{x_{i} \in L_{1}(\mu, Y): x_{i}: \Omega \rightarrow Y\right.$ is or $\vee_{t \in T}$ $\mathscr{F}_{i}{ }^{t}$-measurable and $x_{i}(\omega) \in X_{i}(\omega) \mu$-a.e. $\}$. Also for each $i$, and each $t \in T$, let $L_{x_{1}^{t}}$ be equal to $\left\{x_{i} \in L_{1}(\mu, Y): x_{i}: \Omega \rightarrow Y\right.$ is $\mathscr{F}_{i}^{t}$-measurable and $x_{i}(\omega) \in X_{i}(\omega)$

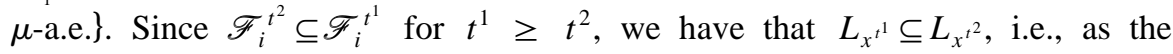
information increases the consumption set of each agent expands. Moreover, for each $t \in T$ we have that $L_{x_{i}^{t}} \subseteq \bar{L}_{x_{i}}$ and by assumption for each $t$ and each $i$, $e_{i}^{t} \in L_{x_{i}^{t}}$ and for each $i, \bar{e}_{i} \in \bar{L}_{x_{i}}$. Notice that if $x^{t} \in L_{x^{t}}$ is a feasible allocation, i.e., $\sum_{i=1}^{n} x_{i}^{t}=\sum_{i=1}^{n} e_{i}^{t}=e^{t}$, then for each $i, 0 \leq x_{i}^{t} \leq e^{t}$. Recall that the space $L_{1}(\mu, Y)$ is a Banach lattice and the martingale $\sum_{i=1}^{n} e_{i}^{t}=e^{t}$ converges to the $L_{1}(\mu, Y)$-norm in $\bar{e}=\sum_{i=1}^{n} \bar{e}_{i}$. By a standard result (e.g., Aliprantis and Burkinshaw, 1985) we can extract a subsequence $e^{t_{k}}: k=1,2, \ldots$ from $\left\{e^{t}: t \in T\right\}$ and also find a positive element $u$ in $L_{1}(\mu, Y)$ such that $\left|e^{t_{k}}-\bar{e}\right|<\frac{1}{2^{k}} u$. Hence, we can conclude that $e^{t_{k}}$ is order bounded above by an element say $v$ in $L_{1}(\mu, Y)$ and clearly below by 0 , i.e., $e^{t_{k}}$ belongs to the order interval $[0, v]$ in $L_{1}(\mu, Y)$. As we noted above for each $i$, and each $t, 0 \leq x_{i}^{t} \leq e^{t}$ and therefore we can conclude that each $x_{i}^{t_{k}}$ lies in the order interval $[0, v]$. Recall that by the Cartwright Theorem the order interval $[0, v]$ is weakly compact. Finally by claim 4.1 in Yannelis (1991) each $v_{i}$ is weakly continuous. With all these preliminary observations out of the way, we are now ready to proceed with the proof.

To this end, for each $t \in T$, let the allocation $x^{t}$ be in $C_{\epsilon}\left(\mathscr{E}_{t}\right)$. Then each $x_{i}^{t}$ is $\mathscr{F}_{i}{ }^{t}$-measurable and by the above construction for each $i$, each subsequence $x_{i}^{t_{m}} \in[0, \nu]$. Let $[0, v]^{n}=[0, v] x \ldots x[0, v]$, i.e., the $n$-fold Cartesian product of $[0, v]$. By the Cartwright Theorem the $[0, v]^{n}$ is weakly compact. Hence, from the subsequence $x^{t_{m}}$ we can obtain a further subsequence still denoted by $x^{t_{m}}$ such that $x^{t_{m}}$ converges weakly to $x^{*} \in[0, v]^{n}$. We need to show that $x^{*}$ is in $C(\overline{\mathscr{E}})$. First note that since $\sum_{i=1}^{n} x_{i}^{t_{m}}=\sum_{i=1}^{n} e_{i}^{t_{m}}, x^{t_{m}}$, converges weakly to $x^{*}$ and $e_{i}^{t_{m}}$ converges in the $L_{1}(\mu, Y)$-norm (and thus weakly) to $\bar{e}_{i}$ (recall assumption a.3.2) we conclude that $\sum_{i=1}^{n} x_{i}^{*}=\sum_{i=1}^{n} \bar{e}_{i}$. Let $\bar{e}=\sum_{i=1}^{n} \bar{e}_{i}$. Since for each $i, x_{i}^{*} \in[0, \bar{e}]$ $\subset \sum_{i=1}^{n} \bar{L}_{x_{i}}$ it follows that each $x_{i}^{*}$ is $\vee_{t \in T} \overline{\mathscr{F}}_{i}^{t}$-measurable. Hence, all that remains to be shown is that:

(5.3.1) there is no coalition $S$ and $y \in \prod_{i \in S} \bar{L}_{x_{i}}$ such that $\sum_{i \in S} y_{i}=\sum_{i \in S} \bar{e}_{i}$ and $v_{i}\left(\omega, y_{i}\right)>v_{i}\left(\omega, x_{i}^{*}\right) \mu$-a.e. for all $i \in S$.

Suppose by way of contradiction that (5.3.1) is not true. Then there exist coalition $S$ and $y \quad y \in \prod_{i \in S} \bar{L}_{x_{i}}$ such that $\left.\left.\sum_{i \in S}\right\} y_{i}=\sum_{i \in S}\right\} \overline{\mathrm{e}}_{i}$ and $v_{i}\left(\omega, y_{i}\right)>$ 
$v_{i}\left(\omega, x_{i}^{*}\right) \mu$-a.e. and for all $i \in S$. For each $i \in S$ and each $t \in T$, let $y_{i}^{t}=E$ $\left[y^{i} \wedge_{i \in S} \mathscr{F}_{i}{ }^{t}\right]$. Notice that

$$
\begin{aligned}
& E\left[y_{i} \bigwedge_{i \in S} \mathscr{F}_{i}{ }^{t}\right]=E\left[E\left[y_{i} \bigwedge_{i \in S} \mathscr{F}_{i}{ }^{t^{\prime}}\right] \mid \bigwedge_{i \in S} \mathscr{F}_{i}{ }^{t}\right] \quad \text { for } t^{\prime} \geq t \\
& =E\left[y_{i}^{t^{\prime}} \bigwedge_{i \in S} \mathscr{F}_{i}{ }^{t}\right] \text { for } t^{\prime} \geq t \text {. }
\end{aligned}
$$

Hence, $\left\{y_{i}^{t}, \wedge_{i \in S} \mathscr{F}_{i}{ }^{t}\right\}_{t \in T}$ is a martingale. Also, It follows from assumption a.3.2 that

$$
\left|\sum_{i \in S} \bar{e}_{i}\right| \bigwedge_{i \in S} \mathscr{F}_{i}{ }^{t}=\sum_{i \in S} e_{i}^{t} .
$$

Hence, $\sum_{i \epsilon S} y_{i}^{t}=\sum_{i \epsilon S} e_{i}^{t}$, i.e., $y^{t}$ is a feasible allocation for the coalition $S$.

By virtue of the martingale convergence theorem $y_{i}^{t}$ converges in the $L_{1}(\mu, Y)$-norm and therefore weakly to $y_{i}$. Since $x^{t}$ also converges weakly to $x^{*}$ and $v_{i}(\omega, \cdot)$ is weakly continuous for each fixed $\omega \in \Omega$, we may choose $t \in T$ so that $\left|v_{i}\left(w, y_{i}\right)-v_{i}\left(w, y_{i}^{t}\right)\right|<\frac{\delta-\varepsilon}{2}$ and $\left|v_{i}\left(w, x_{i}^{t}\right)-v_{i}\left(w, x_{i}^{*}\right)\right|<\frac{\delta-\varepsilon}{2}$ where $\delta=$ $v_{i}\left(\omega, y_{i}\right)-v_{i}\left(\omega, x_{i}^{*}>\varepsilon\right.$. Thus,

$$
\begin{aligned}
& \left|v_{i}\left(w, y_{i}\right)-v_{i}\left(w,,_{i}^{t}\right)+v_{i}\left(w, x_{i}^{t}\right)-v_{i}\left(w, x_{i}^{*}\right)\right| \\
& \quad \leq\left|v_{i}\left(w, y_{i}\right)-v_{i}\left(w, y_{i}^{t}\right)\right|+\left|v_{i}\left(w, x_{i}^{t}\right)-v_{i}\left(w, x_{i}^{*}\right)\right| \\
& \quad<\frac{\delta-\varepsilon}{2}+\frac{\delta-\varepsilon}{2}=\delta-\varepsilon .
\end{aligned}
$$

Therefore, $v_{i}\left(\omega, y_{i}\right)-v_{i}\left(\omega, y i^{t}\right)+v_{i}\left(\omega, x_{i}^{t}\right)-v_{i}\left(\omega, x_{i}^{*}\right)<\delta-\varepsilon \leftrightarrow-v_{i}\left(\omega, y_{i}^{t}\right)$ $+v_{i}\left(\omega, x_{i}^{t}\right)<-\varepsilon$ or $\varepsilon+v_{i}\left(\omega, x_{i}^{t}\right)<v_{i}\left(\omega, y_{i}^{t}\right)$ for all $i \in S$. Consequently, we have shown that the allocation $y^{t}$ is feasible for the coalition $S$, i.e., $\sum_{i \in S} y_{i t}=$ $\sum_{i \epsilon S} e_{i}^{t}$ and $v_{i}\left(\omega, y_{i}^{t}\right)>v_{i}\left(\omega, x_{i}^{t}\right)+\varepsilon \mu$-a.e., and for all $i \in S$, a contradiction to the fact that $x^{t} \in C_{\epsilon}\left(\mathscr{E}^{t}\right)$ for all $t \in T$. Hence, (5.3.1) holds and this completes the proof of the theorem.

Proof of theorem 3.3.2: Let $x$ be an element of $C(\overline{\mathscr{E}})$. Consider the allocation $x^{t}=E\left[x \wedge_{i=1}^{n} \mathscr{F}_{i}^{t}\right]$ and notice that for $r \geq t$

$$
\begin{aligned}
& ] E\left[x \mid \bigwedge_{i=1}^{n} \mathscr{F}_{i}{ }^{t}=E\left[E \left[x\left|\bigwedge_{i=1}^{n} \mathscr{F}_{i}{ }_{i}^{t}\right|_{i=1}^{n} \mathscr{F}_{i}{ }^{t}\right.\right.\right. \\
& ]\left.x^{r}\right|_{i=1} ^{n} \mathscr{F}_{i}{ }_{i} .
\end{aligned}
$$

Hence, $\left\{x^{t}, \wedge{ }_{i=1}^{n} \mathscr{F}_{i}{ }^{t}\right\}_{t \in T}$ is a martingale and by virtue of the martingale convergence theorem $x^{t}$ converges in the $L_{i}(\mu, Y)$-norm to $x$. By the definition of the conditional expectation we know that for each $i, x_{i}^{t}$ is $\mathscr{F}_{i}{ }^{t}$-measurable. We must 
show that the net $\left\{x^{t}: t \in T\right\}$ lies in $C_{\epsilon}\left(\mathscr{E}^{t}\right)$. We first show that $x^{t}$ is feasible for the grand coalition. Note that

$$
\begin{aligned}
\sum_{i=1}^{n} x_{i}^{t}=\sum_{i=1}^{n} E\left[x \mid \bigwedge_{i=1}^{n} \mathscr{F}_{i}{ }_{i}\right] & =E\left[\sum_{i=1}^{n} x_{i} \mid \bigwedge_{i=1}^{n} \mathscr{F}_{i}{ }^{t}\right] \\
& =E\left[\sum_{i=1}^{n} e_{i} \mid \bigwedge_{i=1}^{n} \mathscr{F}_{i}{ }^{t}\right] \\
& =\sum_{i=1}^{n} e_{i}^{t}(\text { recall assumption a.3.4 })
\end{aligned}
$$

and we can conclude that $x^{t}$ is feasible. We now show that the allocation $x^{t}$ cannot be $\varepsilon$-blocked by any coalition, i.e.,

(5.3.2) there does not exist coalition $S$ and allocation $y \in \prod_{i=1}^{n} L_{x_{i}^{t}}=L_{x^{t}}$ such that $\sum_{i \in S} y_{i}=\sum_{i \in S} e_{i}^{t}$ and $v_{i}\left(\omega, y_{i}\right)>v_{i}\left(\omega, x_{i}^{t}\right)+\varepsilon \mu$-a.e. and for all $i \in S$.

Suppose by way of contradiction that (5.3.2) is false for some $t \in T$. Given $\varepsilon>0$, for each coalition $S$, define $K_{S}(\varepsilon)=\left\{t \in T\right.$ : there exists $y^{t} \in \prod_{i \in S} L_{x_{i}^{t}}$ such that $\sum_{i \in S} y_{i}^{t}=\sum_{i \epsilon S} e_{i}^{t}$ and $v_{i}\left(\omega, Y_{i}^{t}\right)>v_{i}\left(\omega, x_{i}^{t}\right)+\varepsilon \mu$-a.e., and for all $\left.i \in S\right\}$. Notice that for $t \in T \backslash \cup{ }_{S \subset I} K_{S}(\varepsilon), x^{t} \in C_{\varepsilon}\left(\mathscr{E}^{t}\right)$. If for each $S \subset I, K_{S}(\varepsilon)$ is a finite set or empty then the set $\left\{x^{t}: t \in T \backslash \cup_{S \subset I} K_{S}(\varepsilon)\right\}$ belongs to $C_{\varepsilon}\left(\mathscr{E}^{t}\right)$ and by construction $x^{t}$ converges (in the $L_{1}(\mu, Y)$-norm) to $x$, in which case the proof is complete. Hence, all we need to show is that $K_{S}(\varepsilon)$ cannot be infinite. To this end suppose that for some $S \cup I$ the set $K_{S}(\varepsilon)$ has an infinite number of elements, then there exists a net $\left\{y^{t}\right\}_{t \in K_{S}}, y^{t} \in \prod_{i \in S} L_{x_{i}^{t}} \subset \prod_{i \in S} \bar{L}_{x_{i}}$ having the property that $\sum_{i \epsilon S} y_{i}^{t}=\sum_{i \epsilon S} e_{i}^{t}$ and $v_{i}\left(\omega, y^{t}\right)>v_{i}\left(\omega, x_{i}^{t}\right)+\varepsilon \mu$-a.e., and for all $i \in S$. Note that by a similar argument as in the proof of the previous theorem we can conclude that for each $t$, each subsequence $y_{i}^{t_{m}}$ lies on the order interval ${ }^{14}[0, v]^{|S|}$ which is weakly compact (recall Cartwright's theorem). Hence, by the weak compactness of $[0, v]^{|S|}$ we can find a subsequence $\left\{y^{t_{r}}\right\}_{t_{r} \in K_{S}}$ so that $y^{t_{r}}$ converges weakly to $y^{*} \in[0, v]^{|S|}$. For this subsequence we have that

$$
\sum_{i \in S} y_{i}^{t_{r}}=\sum_{i \in S} e_{i}^{t_{r}}
$$

Recall that by assumption a.3.3 and the martingale convergence theorem $e^{t}$ converges (in the $L_{1}(\mu, Y)$-norm) and hence weakly to $\bar{e}$. In particular $e^{t_{r}}$ converges weakly to $\bar{e}$. Hence, taking limits in the above expression we obtain that $\left.\sum_{i \in S}\right\} y_{i}=\sum_{i \in S} \overline{\mathrm{e}}_{i}$.

Since $t_{r} \in K_{S}(\varepsilon)$ we have that for each $t_{r}, v_{i}\left(w, y_{i}^{t_{r}}\right)>v_{i}\left(w, x_{i}^{t_{r}}\right)+\varepsilon \mu-$ a.e. and for all $i \in S$. Thus, by the weak continuity of $v_{i}(\omega, \cdot), v_{i}\left(\omega, y_{i}^{*}\right) \geq v_{i}\left(\omega, x_{i}\right)$

\footnotetext{
${ }^{14}$ If $A$ is a set, the symbol $A$ denotes the cardinality of the set $A$, i.e., the number of elements in the set $A$.
} 
$+\varepsilon \mu$-a.e. and for all $i \in S$. Hence, $v_{i}\left(\omega, y_{i}^{*}\right)>v_{i}\left(\omega, x_{i}\right) \mu$-a.e. and for all $i \in S$, i.e., the coalition $S$ qualifies to block $x$, a contradiction to the fact that $x \in C(\overline{\mathscr{E}})$. This completes the proof of theorem 3.3.2.

Lemma 5.1: Assumption a.3.2 is equivalent to:

(a.3.2'), for each coalition $S$ and for each $t, \sigma\left(\sum_{i \in S} e_{i}^{t}\right) \subset \wedge_{i \in S} \mathscr{F}_{i}^{t}$.

Proof: $\left(\right.$ a.3.2 $\left.2^{\prime}\right) \Leftarrow$ (a.3.2). Since for each coalition $S, \sum_{i \in S} e_{i}^{t}, \wedge_{i \in S^{\mathscr{F}}}{ }_{i t \in T}^{t}$ is a martingale we have that:

$$
E\left[\sum_{i \in S} e_{i}^{r} \mid \bigwedge_{i \in S} \mathscr{F}_{i}{ }^{t}\right]=\sum_{i \in S} e_{i}^{t} \text { for all } r \geq t .
$$

By the definition of conditional probability $E\left[\sum_{i \epsilon S} e_{i}^{r} \wedge_{i \epsilon S} \mathscr{F}_{i}^{t}\right]$ is $\left.\wedge_{i \epsilon S}\right\} \mathscr{F}_{i}{ }_{i}$ measurable. Since $\sum_{i \epsilon S} e_{i}^{t}$ is $\sigma\left(\sum_{i \epsilon S} e_{i}^{t}\right)$-measurable it follows that the left-hand side and the right-hand side terms of Eq. (A.1) are $\sigma\left(\sum_{i \epsilon S} e_{i}^{t}\right) \wedge\left(\wedge_{i \epsilon S} \mathscr{F}_{i}^{t}\right)$-measurable. Since $\sigma\left(\sum_{i \epsilon S} e_{i}^{t}\right)$ is the smallest $\sigma$-algebra for which $\sum_{i \in S} e_{i}^{t}$ is measurable, it must be the case that $\sigma\left(\sum_{i \epsilon S} e_{i}^{t}\right) \subset \wedge_{i \in S} \mathscr{F}_{i}{ }^{t}$.

(a.3.2) $\Leftarrow($ a.3.2' $)$. We only need to show that for each coalition $S,\left\{\sum_{i \in S} e_{i}^{t}\right.$, $\left.\sigma\left(\sum_{i \in S} e_{i}^{t}\right)\right\}_{t \in T}$ is a martingale. Indeed, since $\left.\sigma\left(\sum_{i \epsilon S} e_{i}^{t}\right) \subset \wedge_{i \in S}\right\} \mathscr{F}_{i}^{t}$ it follows that $\left\{\sum_{i \in S} e_{i}^{t}, \wedge_{i \in S} \mathscr{F}_{i}{ }^{t}\right\}_{t \in T}$ is a martingale as well. Observe that for $r \geq t$ :

$$
\begin{aligned}
E\left[\sum_{i \in S} e_{i}^{r} \mid \sigma\left(\sum_{i \in S} e_{i}^{t}\right)\right] & =\sum_{i \in S} E\left[e_{i}^{r} \mid \sigma\left(\sum_{i \in S} e_{i}^{t}\right)\right] \\
& =\sum_{i \in S} E\left[E\left[e_{i}^{r} \mid \mathscr{F}_{i}^{t}\right] \mid \sigma\left(\sum_{i \in S} e_{i}^{t}\right)\right] \\
& =\sum_{i \in S} E\left[e_{i}^{t} \mid \sigma\left(\sum_{i \in S} e_{i}^{t}\right)\right]\left(\text { recall that } E\left[e_{i}^{r} \mid \mathscr{F}_{i}{ }_{i}\right]=e_{i}^{t}\right) . \\
& =E\left[\sum_{i \in S} e_{i}^{t} \mid \sigma\left(\sum_{i \in S} e_{i}^{t}\right)\right] \\
& =\sum_{i \in S} e_{i}^{t}
\end{aligned}
$$

Hence, $\left\{\sum_{i \in S} e_{i}^{t}, \sigma\left(\sum_{i \in S} e_{i}^{t}\right)\right\}_{t \in T}$ is a martingale.

The proofs of theorems 4.2.1 and 4.2.2 can follow by mimicking the arguments of theorems 3.3.1 and 3.3.2, respectively. (The reader must recall that the Shapley value of each agent $i$, i.e., $\mathrm{Sh}_{i}\left(V_{\lambda}\right)$ is continuous in $\lambda$.) In order to avoid repetition we do not provide the straightforward details. 


\section{Acknowledgements}

We wish to thank the referees for their comments and most importantly Roko Aliprantis and Konstantinos Serfes. Moreover, we would like to thank for comments, discussions, and suggestions the participants in the Mathematical Economics Conference at the Math. Institute in Oberwlfach, at the NBER Conference on Decentralization in Toronto and the Workshop on Geometry, Topology and Markets at the Fields Institute in Waterloo. Early versions of this paper were presented in departmental seminars at Caltech, Columbia University, Johns Hopkins University, New York University, the University of Pennsylvania, Penn State University and Texas A\&M University. We benefited from questions and suggestions by participants at all of these institutions. As always we are responsible for any shortcomings. The financial support of the University of Illinois at UrbanaChampaign Campus Research Board is gratefully acknowledged.

\section{References}

Aliprantis, C.D., Burkinshaw, O., 1985. Positive Operators, Academic Press, New York, London. Aliprantis and Burkinshaw, 1989.

Aumann, R.J., 1987. Correlated equilibria as an expression of Bayesian rationality. Econometrica 55, $1-18$.

Cartwright, D., 1974. The order completeness of some vector-valued functions. Bull. Aust. Math. Soc. $11,57-61$.

Diestel, J., Uhl, J.J., 1977. Vector Measures, Mathematical Surveys, 15, Am. Math. Soc., Providence, RI.

Feldman, M., 1987. An example of converge to rational expectations with heterogeneous beliefs. Int. Econ. Rev. 28, 635-650.

Gale, D., 1992. A Walrasian theory of markets with adverse selection. Rev. Econ. Studies 59, 229-255.

Grodal, B., 1971. A theorem on correspondences and continuity of the core. In: Kuhn, H., Szego, G. (Eds.), Game Theory and Related Topics, North Holland, pp. 221-233.

Kalai, E., Lehrer, E., 1993. Rational learning leads to Nash equilibrium. Econometrica 61, 1019-1045.

Krasa, S., Yannelis, N.C., 1994. The value allocation of an economy with differential information. Econometrica 62, 881-900.

Krasa, S., Yannelis, N.C., 1996. Existence and properties of a value allocation for an economy with differential information. J. Math. Econ. 25, 165-179.

Koutsougeras, L., Yannelis, N.C., 1993. Incentive compatibility and information superiority of the core of an economy with differential information. Econ. Theory 3, 195-216.

Koutsougeras, L., Yannelis, N.C., 1994. Convergence and approximation results for non-cooperative Bayesian games: learning theorems. Econ. Theory 4, 843-858.

Kurz, M., 1994. On the structure and diversity of rational beliefs. Econ. Theory 4, 877-900.

Myerson, R.B., 1984. Cooperative games with incomplete information. Int. J. Game Theory 13, 69-96. Nyarko, Y., 1994. Bayesian learning in repeated games leads to correlated equilibria. Econ. Theory 4, $821-842$.

Radner, R., 1968. Equilibrium under uncertainty. Econometrica 36, 31-58.

Shapley, L.A., 1953, A value of $n$-person games. In: Kuhn, H.W., Tucker, A.W. (Eds.), Contributions to the Theory of Games, Vol. II, Princeton Univ. Press, pp. 307-317.

Yannelis, N.C., 1991. The core of an economy with differential information. Econ. Theory 1, 183-198. 